\title{
Clinical Trial Report: Eradication of Helicobacter pylori Reduces the Risk for Subsequent Gastric Cancer
}

\author{
Joseph R. Pisegna • Bijal Surti • David R. Scott
}

Published online: 22 September 2010

(C) The Author(s) 2010. This article is published with open access at Springerlink.com

Fuccio L, Zagari RM, Eusebi LH, et al.: Meta-analysis: can Helicobacter pylori eradication treatment reduce the risk for gastric cancer? Ann Intern Med 2009, 151:121128.

\section{Rating}

-Of importance.

\section{Introduction}

Helicobacter pylori is a carcinogen and has been associated with the development of gastric cancer. The hypothesis to be tested in this meta-analysis is to determine whether eradication of this organism is associated with a long-term reduction in the risk for development of gastric cancer.

J. R. Pisegna $\cdot$ B. Surti $\cdot$ D. R. Scott

CURE: Digestive Diseases Research Center, VA Greater Los Angeles Healthcare System,

Los Angeles, CA 90073, USA

\section{J. R. Pisegna $\cdot$ B. Surti $\cdot$ D. R. Scott}

Department of Medicine, University of California, Los Angeles,

Los Angeles, CA 90073, USA

J. R. Pisegna $(\bowtie)$

Division of Gastroenterology and Hepatology, VA Greater Los

Angeles Healthcare System,

11301 Wilshire Boulevard,

Los Angeles, CA 90073, USA

e-mail: jpisegna@ucla.edu

\begin{abstract}
Aims
The study's purpose is to examine whether eradication of H. pylori infection can reduce the risk for the development of gastric cancer.
\end{abstract}

\section{Methods}

The authors examined the results of seven studies that met inclusion criteria by using relevant clinical trials. Relevant trials were identified through searching PubMed, Embase, Google Scholar, and the Cochrane Library. To be eligible to include subjects in this analysis, the randomized clinical trials were required to compare an eradication treatment group to an untreated group and to provide an analysis of the number of gastric cancers occurring during follow-up evaluation.

\section{Results}

A total of seven studies were considered adequate to meet the inclusion criteria. One of the studies was excluded after further analysis. Overall, 27 of $3388(1.1 \%)$ treated patients in the $H$. pylori antibiotic treatment group were identified as having gastric cancer, compared to 56 of 3307 (1.7\%) in those subjects who did not undergo treatment. The authors identified the relative risk for gastric cancer as $0.65(95 \%$ CI, 0.43-0.98).

\section{Discussion}

The authors conclude that eradication of $H$. pylori reduces gastric cancer risk. 


\section{Comments}

This section compares the meta-analysis under discussion with other studies that evaluate the role of treatment of $H$. pylori infection in reference to the potential positive effects on prevention of gastric cancer. These studies collectively provide the reader with perspective when confronted with a patient with an active infection.

The current article evaluates the protective effects of treating $H$. pylori on the potential risk for the later development of gastric cancer. This article has worldwide impact, given the high incidence of this infection in developing nations, particularly in Asia. This metaanalysis has accordingly focused on enrolling studies from areas of the world that have a high incidence of infection. Although this focus could be viewed as a possible weakness of the inclusion study design, the inclusion of studies from areas with high disease incidence make this study more relevant. This study is supported by a clinical trial evaluating early $H$. pylori eradication in reducing the risk of gastric cancer in patients with peptic ulcer disease [1]. As a corollary, one study showed that $H$. pylori infection is associated with a reduced risk of esophageal cancer [2].

H. pylori is a bacterium that colonizes the relatively acidic human stomach. Like Escherichia coli, it is a neutralophile, an organism that requires nearly neutral $\mathrm{pH}$ for optimal growth. H. pylori has evolved acid acclimation and resistance mechanisms to combat gastric acidity, which permits the colonization of the acidic gastric mucosa. A major adaptation of the organism to the acidic gastric environment is the constitutive production of large amounts of intrabacterial urease [3]. Activation of urease at acidic $\mathrm{pH}$ is a result of the opening of the proton-gated urea channel, UreI. Opening this channel allows rapid entry of urea into the cytoplasm of the organism, where it is hydrolyzed by urease and the production of $\mathrm{NH}_{3}$ and $\mathrm{CO}_{2}$.

H. pylori colonization, if left untreated, results in a persistent, lifelong infection [4, 5]. In developing nations, the incidence of infection is greater than $80 \%$ in adults, whereas industrialized countries report an incidence of 20 $50 \%$. Immigration of persons from endemic areas of the world to the United States results in regional increases in the incidence of disease. Accordingly, H. pylori infection will remain endemic in the United States for at least another century [6]. It is estimated that $15-20 \%$ of patients with $H$. pylori infection develop peptic ulcers and $3 \%$ of infected patients develop gastric cancer [7]. The two primary forms of gastric cancer that have been associated with $H$. pylori infection include adenocarcinoma and, less commonly, mucosa-associated lymphoid tumors (MALToma) or lymphomas [4]. Eradication of $H$. pylori in patients with duodenal or gastric ulcer cures the disease, prevents relapse, and reduces the risk of cancer [6-8].

The diagnosis of $H$. pylori infection can be established by several methods: endoscopy with biopsy, serum antibody testing, urea breath testing, and, more recently, stool antigen assay. During endoscopy, the biopsy results may be confirmed by one of three methods: biopsy, urease test, and histology. Although gastric biopsy is usually considered the gold standard, more studies now rely on less invasive testing. Accordingly, noninvasive testing is the preferred method for establishing a diagnosis and confirming eradication. The sensitivity of urea breath testing is about $88-95 \%$, and specificity is about 95 $100 \%$ [9]. Another method of establishing the diagnosis is laboratory-based serologic testing using enzyme-linked immunosorbent assay technology to detect IgG antibodies. Although this test has a uniform high sensitivity (90\%-100\%), the variable specificity $(76 \%-96 \%)$ and low accuracy (83\%-98\%) would exclude this methodology for confirming eradication. The presence of $H$. pylori in the stool of infected patients has led to the development of a stool antigen test, which is now a commercially available enzyme immunoassay (Premier Platinum HpSA Immunoassay; Meridian Diagnostics, Cincinnati, $\mathrm{OH}$ ), thus enabling this method as an accurate modality for establishing eradication [10]. The accuracy of the test was evaluated in a study involving 270 patients in whom the diagnosis of $H$. pylori was established by endoscopy and urea breath testing; test sensitivity was $94 \%$ and specificity was $86 \%$. Thus, it has been well established that the stool antigen assay appears to be useful for documenting whether eradication was successful. Furthermore, the stool antigen assay is a noninvasive test that, unlike endoscopic tests, does not place patients at risk of developing complications. A 2007 guideline from the American College of Gastroenterology recommends that biopsy for histopathology is only appropriate if urea breath testing or stool antigen testing is not feasible or during follow-up of complicated ulcer disease, and that serologic testing is not useful for follow-up because many patients continue to have antibodies for months or years after eradication therapy [11]. In the meta-analysis under review, four of the clinical studies relied on urea breath testing and two of the studies relied on the rapid urease test. Histology was used for confirmation in three of the studies.

H. pylori bacterial infection is prevalent in certain US populations, and leads to the development of peptic ulcer disease and a predisposition to gastric cancer [2]. Eradication of $H$. pylori prevents peptic ulcer disease and reduces the risk of gastric cancer, and thus is an important therapeutic strategy for most clinicians [1, 6-8]. Current $H$. pylori eradication therapy requires a combination treatment approach using a proton pump inhibitor (PPI) 
and at least two antibiotics. This approach is known as "triple therapy." Standard eradication therapy requires treatment for 10-14 days and has a success rate of less than $75 \%$ [12]. The efficacy of various triple-therapy regimens is undermined by the development of antimicrobial resistance by $H$. pylori [13] and may well contribute to development of antibiotic resistance of other important bacterial pathogens [14]. In $H$. pylori infection, primary resistance to amoxicillin has not been described. However, the frequency of clarithromycin resistance is $17-45.4 \%$ in the United States and even higher in Japan. It is estimated that the resistance rate worldwide is increasing. Metronidazole resistance ranges between $25 \%$ and $48 \%$ in both men and women in developing countries, because of the frequent use of nitro-imidazoles to treat other diseases [15]. In a recent meta-analysis, primary resistance to clarithromycin decreased the eradication rate by $50 \%$, whereas primary resistance to metronidazole decreased the rate of eradication by $37 \%$ [16]. The rate of eradication using the triple-therapy approach has fallen below $80 \%$ in many regions, as noted in the Maastricht III consensus report, and the results differ by regions of the world [17]. The development of antimicrobial resistance by $H$. pylori is a major factor in unsuccessful eradication, and clarithromycin treatment may contribute to development of antibiotic resistance of other important pathogens $[13,14$, $18,19]$. Antibiotic therapy is associated with diarrhea and the potential for the development of pseudomembranous colitis. Poor patient compliance is also a factor in unsuccessful eradication. Twice-daily administration of a PPI and twice-daily administration of two antibiotics for 14 days requires 84 tablets; moreover, compliance with before-meal administration is necessary because of the acid secretion-dependent mechanism of the PPI. Additionally, PPI-based acid control has considerable variability because of variability in absorption and drug-drug interactions owing to metabolism by CYP2C19, which may account for the lack of universal eradication with triple therapy. Although the meta-analysis reviewed here does not specifically address antimicrobial resistance, this issue is critically important and its omission may result in this study underestimating the potential relative risk reduction of the development of gastric cancer. The importance of eradication is not only for the prevention and cure of peptic ulcer disease, but also for the prevention of gastric adenocarcinoma, which is the third leading cause of cancer deaths in the United States.

Overall, the available data suggest that $H$. pylorus is a carcinogen and in certain persons leads to the development of gastric cancer. Although clinical and epidemiologic studies show an increase in relative risk for the development of gastric cancer in individuals infected with this organism, the exact mechanism that explains the link between this organism and carcinogenesis is not known. Thus, a review of the available literature indicates strong clinical evidence suggesting that successful eradication of this organism is related to a reduction in the risk of gastric cancer. Clearly, a better understanding of this pathophysiological association is needed to better define the pathways involved in carcinogenesis.

Disclosure No potential conflict of interest relevant to this article was reported.

Open Access This article is distributed under the terms of the Creative Commons Attribution Noncommercial License which permits any noncommercial use, distribution, and reproduction in any medium, provided the original author(s) and source are credited.

\section{References}

1. Wu C-Y, Kuo KN, Wu M-S, et al.: Early Helicobacter pylori eradication decreases risk of gastric cancer in patients with peptic ulcer disease. Gastroenterology 2009, 137:1641-1648.

2. Whireman DC, Parmar P, Fahey P, et al.: Association of Helicobacter pylori infection with reduced risk for esophageal cancer is independent of environmental and genetic modifiers. Gastroenterology 2010, 139:73-83.

3. Mobley HL, Island MD, Hausinger RP: Molecular biology of microbial ureases. Microbiol Rev 1995, 59:451-480.

4. Blaser MJ: Helicobacter pylori and gastric diseases. BMJ 1998, 316:1507-1510.

5. Blaser MJ, Atherton JC: Helicobacter pylori persistence: biology and disease. J Clin Invest 2004, 113:321-333.

6. Uemura N, Okamoto S, Yamamoto S, et al.: Helicobacter pylori infection and the development of gastric cancer. N Engl J Med 2001, 345:784-789.

7. Uemura N, Mukai T, Okamoto S, et al.: Effect of Helicobacter pylori eradication on subsequent development of cancer after endoscopic resection of early gastric cancer. Cancer Epidemiol Biomarkers Prev 1997, 6:639-642.

8. Akre K, Signorello LB, Engstrand L, et al.: Risk for gastric cancer after antibiotic prophylaxis in patients undergoing hip replacement. Cancer Res 2000, 60:6376-6380.

9. Howden CW, Hunt RH: Guidelines for the management of Helicobacter pylori infection. Am J Gastroenterol 1998, 93:2330

10. Trevisani L, Sartori S, Galvani F, et al.: Evaluation of a new enzyme immunoassay for detecting Helicobacter pylori in feces: a prospective pilot study. Am J Gastroenterol 1999, 94:1830.

11. Chey WD, Wong BCY: American College of Gastroenterology guideline on the management of Helicobacter pylori infection. Am J Gastroenterol 2007, 102:1808.

12. Padol S, Yuan Y, Thabane M, et al.: The effect of CYP2C19 polymorphisms on $H$. pylori eradication rate in dual and triple first-line PPI therapies: a meta-analysis. Am J Gastroenterol 2006, 101:1467-1475.

13. Graham DY: Antibiotic resistance in Helicobacter pylori: implications for therapy. Gastroenterology 1998, 115:1272-1277.

14. Megraud F. Resistance of Helicobacter pylori to antibiotics. Aliment Pharmacol Ther 1997, 11(Suppl 1):43-53. 
15. Boyanova L, Gergova G, Nikolov R, et al.: Prevalence and evolution of Helicobacter pylori resistance to 6 antibacterial agents over 12 years and correlation between susceptibility testing methods. Diagn Microbiol Infect Dis 2008, 60:409415.

16. Dore MP, Leandro G, Realdi G, et al.: Effect of pretreatment antibiotic resistance to metronidazole and clarithromycin on outcome of Helicobacter pylori therapy: a meta-analytical approach. Dig Dis Sci 2000, 45:68-76.
17. Malfertheiner P, Megraud F, O'Morain C, et al.: The European Helicobacter Study. Current concepts in the management of Helicobacter pylori infection: the Maastricht III Consensus Report. Gut 2007, 56:772-781.

18. Megraud F: Helicobacter pylori and antibiotic resistance. Gut 2007, 56:1502.

19. Penston JG, McColl KE: Eradication of Helicobacter pylori: an objective assessment of current therapies. Br J Clin Pharmacol 1997, 43:223-243. 